\title{
Tandospirone, a 5-HT1A partial agonist is effective in treating anorexia nervosa: a case series
}

\author{
Kyoji Okita ${ }^{1 *}$, Akihiro Shiina ${ }^{1,2}$, Michiko Nakazato ${ }^{1,3}$ and Masaomi lyo ${ }^{1,2}$
}

\begin{abstract}
This case report details the therapeutic effects of tandospirone on two patients with anorexia nervosa, one with the restricting subtype (ANR), and another with the binge-eating/purging subtype (ANBP). A 22-year-old female patient with ANR and a 23-year-old female patient with ANBP were treated successfully with the 5-HT1A partial agonist tandospirone. After treatment, not only did both patients gain weight, they also showed improved scores on the Eating Disorder Examination Questionnaire. In addition, a 6-month follow-up showed maintenance of these effects. In this disorder, tandospirone may be an effective drug for long-term use with good patient compliance.
\end{abstract}

Keywords: Anorexia nervosa, Pharmacotherapy, Treatment, Serotonin 1A receptor, Tandospirone

\section{Introduction}

Eating disorders such as anorexia nervosa (AN) have a relatively high incidence in women and the highest mortality rate among mental disorders [1]. Patients suffering from AN show characteristic symptoms, typified by an intense fear of gaining weight or becoming fat, body image distortion, and eating concerns. They are distressed by their obsessive psychopathology. Even when their body mass index falls below minimum levels, they still report feeling larger than they actually are and maintain their fear of gaining weight. Although they have a keen interest in eating, they often restrict calorie consumption and/or vomit after binge eating. In addition, despite significant physical symptoms, patients tend to be in self-denial about the seriousness of their disease. Attempts to treat this disease are confounded by patients frequently presenting with physical problems, such as heart failure, increased susceptibility to infection, and osteoporosis.

There are currently no definitively effective treatments for AN, and although various medications including selective serotonin reuptake inhibitors (SSRIs) or antipsychotics have been evaluated in the past, there is little evidence to support the benefit of psychotropic medication for AN treatment [2]. One reason for this lack of evidence is that robust, randomized, placebo-controlled studies are difficult

\footnotetext{
* Correspondence: okitak@graduate.chiba-u.jp

'Department of Psychiatry, Graduate School of Medicine, Chiba University,

1-8-1 Inohana, Chuo-ku, Chiba, Chiba 260-8677, Japan

Full list of author information is available at the end of the article
}

to perform due to high dropout rates [3]. This lack of compliance may be due to denial about their disease as well as a continued fear of gaining weight. Treatments that target the fear of gaining weight and body image distortion seem to be more acceptable to AN patients and therefore more effective than treatments that solely target weight gain. This is a pivotal issue for consideration in the prevention of relapse and aggravation of symptoms [4].

There are several biological hypotheses about AN that highlight a relationship between disease pathology and serotonin [5]. Neuroimaging studies (e.g., positron emission tomography and single photon emission tomography) have identified a role for the 5-HT1A receptor, and it is already known that upregulated expression of this receptor in various areas of the brain is associated with anxiety [6].

This report aims to show the effects of the 5-HT1A partial agonist tandospirone in the treatment of AN. Both cases showed weight gain in a relatively short time period and improvement in the Eating Disorder Examination Questionnaire (EDE-Q) [7], which is a self-report questionnaire useful for determining the effect of treatment and providing a comprehensive assessment of the behavioral and psychological parameters characteristic of the disorder. The EDE-Q generates four subscales (restraint, eating concern, weight concern, and shape concern) as well as a global score. Higher scores indicate a greater severity of eating disorder psychopathology.

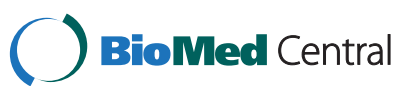




\section{Case presentation Case A}

Patient A is a 23-year-old married female with one child. After her husband had an affair with a thinner woman, she decided to lose weight. Subsequent to this, she began to experience nausea and palpitations on eating. Before these symptoms developed, she weighed approximately $50 \mathrm{~kg}$, with a body mass index (BMI) of 20.6. Within 5 months, she weighed $41 \mathrm{~kg}$ and was amenorrheic. Her eating patterns were severely restricted to one meal between morning and noon per day.

She presented at our clinic complaining of noncausal nausea and weight loss. At her first visit, she measured $158-\mathrm{cm}$ tall and weighed $41.3 \mathrm{~kg}$, with a BMI of 16.5. At this point, she had already lost $8 \mathrm{~kg}$ and had not had menses for almost 4 months. Although she admitted that she had lost weight, she felt that she still looked the same and expressed a desire to reduce her weight further to $30 \mathrm{~kg}$. She also complained about a fear of gaining weight. She was diagnosed in accordance with DSM-IV-TR as having AN of restricted type (ANR).

She was prescribed $1 \mathrm{mg}$ of ethyl lofrazepate to combat her anxiety attacks and palpitations. While this symptom showed small improvement, she maintained her fear of weight gain and eating patterns and showed further weight loss from 41.3 to $40 \mathrm{~kg}$ (BMI 16.4). At a weight of $40.0 \mathrm{~kg}$, her EDE-Q subscales of restricting/ eating concern/weight concern/shape concern and global scores were 4.6/1.6/3.6/4.88 and 3.67, respectively. She was started on tandospirone because of its fewer side effects on the digestive system relative to SSRIs and its antianxiety effects. Patient A was scheduled for two weekly visits to our clinic to monitor side effects, although no specific psychological treatment was provided. At these visits and in the absence of side effects, tandospirone was initiated at $20 \mathrm{mg}$ and escalated by 20 mg every 2 weeks until it reached $60 \mathrm{mg}$. It was recommended that she take tandospirone twice daily (when prescribed 20 and $40 \mathrm{mg}$ ) or three times daily (when prescribed $60 \mathrm{mg}$ ). From the onset of treatment, our patient's dietary intake gradually increased, and she began to gain weight. In addition, she no longer complained about feelings of fear and guilt. Four weeks into therapy, her weight had increased to $43 \mathrm{~kg}$ (BMI 17.7), and her EDE-Q scores reduced, with the subscale scores of restricting/eating concern/weight concern/ shape concern and global score being 3.4/0.6/1.2/4.0 and 2.3, respectively. After approximately 20 weeks, her menses resumed. At the 6-month point, she was still adhering to her treatment regime, her EDE-Q diagnostic score was 6, and the subscales restricting/eating concern/weight concern/shape concern and global score were 1.2/0/2.0/3.0 and 1.55, respectively. Also, she had gained weight to reach $45.0 \mathrm{~kg}$ with a BMI of 18.5 .

\section{Case B}

Patient B is a 22-year-old female. She began to restrict her eating after gaining $20 \mathrm{~kg}$ during pregnancy. Soon after this, she drank only water during the day and overate and purged in the evening. Within 6 months, she became amenorrheic.

She presented at our hospital seeking help to curb her binge eating and purging. She was in a pattern of binge eating and purging twice in a day. She felt depressed and irritated that she was not in a good physical condition and unable to be a good parent. At the first visit, she measured 153-cm tall, weighed $35.0 \mathrm{~kg}$, and had a BMI of 15.0. Although she had already experienced a $13-\mathrm{kg}$ weight loss and almost 10 months of amenorrhea, she displayed body image distortion saying 'If I lose more weight, I will be fine.' Additionally she stated 'I feel more confident when losing weight.' It was clear that her self-esteem was tightly linked to her weight. She was diagnosed according to DSM-IV-TR with AN of binge/ purging type (ANBP).

First, she was prescribed $1 \mathrm{mg}$ of ethyl lofrazepate to improve her irritability. This proved effective for her insomnia, but her purging habits gradually deteriorated, and she began cathartic abuse. As a result, her weight fell to $34.8 \mathrm{~kg}$ (BMI 14.9). At this weight, the EDE-Q subscales of restricting/eating concern/weight concern/ shape concern and global score were 2.4/4.4/4.2/5.25 and 4.06, respectively. Tandospirone was started at 20 $\mathrm{mg}$ and escalated by $20 \mathrm{mg}$ at 2 -week intervals until it reached $60 \mathrm{mg}$, as in case A. Four weeks after the initiation of tandospirone, she felt an improvement in her depressive mood, and her weight increased to $36.3 \mathrm{~kg}$ (BMI 15.4). At this time, the EDE-Q subscales of restricting/eating concern/weight concern/shape concern and global score were 2.4/2.2/0.2/1.75 and 1.64, respectively. Although her binge eating and purging continued, she stopped cathartic abuse 3 months into treatment and reduced her binge eating and purging to once a day. Her weight increased further to $39.0 \mathrm{~kg}$ (BMI 16.7) by week 24 , and her EDE-Q subscales of restricting/eating concern/weight concern/shape concern and global score were $2.4 / 2.0 / 0.6 / 3.5$ and 2.13, respectively. Shape concern and weight concern subscale scores increased compared to those obtained in week 4 . This is thought to be due to the subject being in a bad mood after a patient commented that she looked fatter.

\section{Discussion}

To the best of our best knowledge, this is the first case report showing a therapeutic effect of tandospirone in treating $\mathrm{AN}$ of both the restricting and binge/purging types. In the USA and Europe, an equivalent 5-HT1A receptor partial agonist, buspirone, is available on the market, but its effect in treating $\mathrm{AN}$ is unknown. 
Several studies using positron emission tomography and single photon emission computed tomography with 5-HT-specific radioligands have consistently shown 5HT1A transporter alterations in AN patients within the cortical and limbic structures, which may be related to behavioral inhibition and body image distortions [5]. Tandospirone mediates two important effects that are integral to alleviating the symptoms of AN. First, tandospirone induces improvement not only in weight gain but also in EDE-Q scores, suggesting improved psychopathology of the patient towards their disorder (Table 1). Disease psychopathology is a core and treatment-refractive symptom of AN. Patients have a morbid fear of weight gain and body image distortion. Treatments that target this core symptom are more likely to be effective in terms of patient compliance and thus prevention of relapse and aggravation of symptoms, compared with treatments that only target weight gain. In these two cases, only psychotropic medication was administered, and no AN-specific psychotherapy was performed. We found that tandospirone improved EDEQ scoring in both patients. Significant improvements were seen in shape concern and global scores, when comparing week 0 with week 24 , using $t$ test (Table 1 ). After treatment, patient A was able to eat an additional afternoon meal, while patient B stopped her cathartic abuse and reduced her binge and purge behavior to once a day. As mentioned above, PET studies have shown that 5-HT1A receptors are highly expressed in specific areas of the brain in patients with AN. This high expression in the dorsal raphe leads to a deterioration of serotonin neuron activity, resulting in a decrease of extracellular serotonin [8]. Tandospirone is a selective agonist of the 5-HT1A receptor [9], and it is thought to desensitize presynaptic 5-HT1A autoreceptors on dendrites of serotonin neurons in the dorsal raphe and thus normalize the function of serotonin neurons [10]. It is also possible that tandospirone may act directly on the 5-HT1A autoreceptor to improve the psychopathology seen in AN.

Secondly, tandospirone treatment has a low incidence of side effects. Patients with AN often also suffer from a range of physical problems, such as loss of bone mass or severe obstipation, as a consequence of being in a persistent state of subnutrition. Therefore, the low incidence of side effects, particularly digestive symptoms that interfere with eating or hypnesthesia that may increase the risk of falls, does not add to the patient's burden. Pharmacologically, tandospirone has a much lower frequency of side effects than the majorly prescribed SSRIs or antipsychotics used in Japan [11-15]. Antipsychotic medication has been proven useful in treating AN [16], but the duration of these studies is relatively short, and the efficacy for relapse prevention in the long term is not proven. In addition, the associated weight gain induced by these drugs leads to high levels of patient noncompliance. To date, there is no evidence that tandospirone induces weight gain. These combined factors make tandospirone an attractive drug for long-term use, with high compliance in this cohort of patients.

This study is by no means conclusive. The relapse rate of $\mathrm{AN}$ is very high, and in this study, our patients were followed up for 6 months. This time frame may need to be extended in order to evaluate the effect of recurrence prevention with this drug. Further, controlled trials in AN are needed to fully explore the effects of tandospirone in clinical practice.

\section{Conclusion}

We report cases of patients with ANR and ANBP who were successfully treated with 5-HT1A partial agonist tandospirone. Tandospirone may be an effective drug for long-term use with good patient compliance due to its pharmacological characteristics.

Table 1 Progress in BMI and the EDE-Q of the two cases since administering tandospirone

\begin{tabular}{|c|c|c|c|c|c|c|c|}
\hline & \multicolumn{6}{|c|}{ Time after tandospirone (weeks) } & \multirow[t]{3}{*}{$p$ value $^{a}$} \\
\hline & \multicolumn{3}{|c|}{ Case A } & \multicolumn{3}{|c|}{ Case B } & \\
\hline & 0 & 4 & 24 & 0 & 4 & 24 & \\
\hline BMl & 16.4 & 17.7 & 18.5 & 14.9 & 15.4 & 16.7 & ${ }^{*} p=0.049$ \\
\hline Height & 156 & 156 & 156 & 153 & 153 & 153 & \\
\hline Weight & 40.0 & 43.0 & 45.0 & 34.8 & 36.3 & 39.0 & \\
\hline \multicolumn{8}{|l|}{ EDE-Q } \\
\hline Restricting subscale & 4.6 & 3.4 & 1.2 & 2.4 & 2.4 & 2.4 & $p=0.500$ \\
\hline Eating concern subscale & 1.6 & 0.6 & 0.0 & 4.4 & 2.2 & 2.0 & $p=0.126$ \\
\hline Weight concern subscale & 3.6 & 1.2 & 2.0 & 4.2 & 0.2 & 0.6 & $p=0.234$ \\
\hline Shape concern subscale & 4.88 & 4.0 & 3.0 & 5.25 & 1.75 & 3.5 & ${ }^{*} p=0.023$ \\
\hline Global score & 3.67 & 2.3 & 1.55 & 4.06 & 1.64 & 2.13 & ${ }^{*} p=0.028$ \\
\hline
\end{tabular}

a Paired Student's $t$ test, two-tailed alpha level of 0.05 at 0 week versus 24 week. ${ }^{*} p<0.05$. 


\section{Consent}

Written informed consents were obtained from the patients for publication of this case series and accompanying images. A copy of the written consent is available for review by the Editor-in-Chief of this journal.

\section{Abbreviations}

AN: anorexia nervosa; ANR: anorexia nervosa restricting subtype;

ANBP: anorexia nervosa binge-eating/purging subtype.

\section{Competing interests}

The authors declare that they have no competing interests.

\section{Authors' contributions}

$\mathrm{KO}, \mathrm{AS}, \mathrm{MN}$, and $\mathrm{MI}$ contributed equally during the clinical evaluation and follow-up period. $\mathrm{KO}$ wrote the first draft of the manuscript. Ml is the principal investigator of this study. All authors contributed to and have read and approved the final manuscript.

\section{Author details}

'Department of Psychiatry, Graduate School of Medicine, Chiba University, 1-8-1 Inohana, Chuo-ku, Chiba, Chiba 260-8677, Japan. ${ }^{2}$ Center for Forensic Mental Health, Chiba University, 1-8-1 Inohana, Chuo-ku, Chiba, Chiba2608670, Japan. ${ }^{3}$ Research Center for Child Mental Development, Graduate School of Medicine, Chiba University, 1-8-1 Inohana, Chuo-ku, Chiba, Chiba 260-8670, Japan.

Received: 11 December 2012 Accepted: 4 March 2013

Published: 9 March 2013

\section{References}

1. Arcelus J, Mitchell AJ, Wales J, Nielsen S: Mortality rates in patients with anorexia nervosa and other eating disorders. A meta-analysis of 36 studies. Arch Gen Psychiatry 2011, 68:724-731.

2. Hay PJ, Claudino AM: Clinical psychopharmacology of eating disorders: a research update. Int J Neuropsychopharmacol 2012, 15:209-222.

3. Fazeli PK, Calder GL, Miller KK, Misra M, Lawson EA, Meenaghan E, Lee H, Herzog D, Klibanski A: Psychotropic medication use in anorexia nervosa between 1997 and 2009. Int J Eat Disord 2012, 45(8):970-976.

4. Hebebrand J: Pharmacotherapy of anorexia nervosa: more questions than answers. J Am Acad Child Adolesc Psychiatry 2011, 50:854-856.

5. Bailer UF, Kaye WH: Serotonin: imaging findings in eating disorders. Curr Top Behav Neurosci 2011, 6:59-79.

6. Galusca B, Costes N, Zito NG, Peyron R, Bossu C, Lang F, Le Bars D, Estour B: Organic background of restrictive-type anorexia nervosa suggested by increased serotonin $1 \mathrm{~A}$ receptor binding in right frontotemporal cortex of both lean and recovered patients: [18 F]MPPF PET scan study. Biol Psychiatry 2008, 64:1009-1013.

7. Luce KH, Crowther JH: The reliability of the Eating Disorder ExaminationSelf-Report Questionnaire Version (EDE-Q). The International Journal Of Eating Disorders 1999, 25:349-351.

8. Pineyro G, Blier P: Autoregulation of serotonin neurons: role in antidepressant drug action. Pharmacol Rev 1999, 51:533-591.

9. Hamik A, Oksenberg D, Fischette C, Peroutka SJ: Analysis of tandospirone (SM-3997) interactions with neurotransmitter receptor binding sites. Biol Psychiatry 1990, 28:99-109.

10. Godbout R, Chaput Y, Blier P, de Montigny C: Tandospirone and its metabolite, 1-(2-pyrimidinyl)-piperazine-I. Effects of acute and long-term administration of tandospirone on serotonin neurotransmission. Neuropharmacology 1991, 30:679-690.

11. Pharma DS: Interview form [Sediel ${ }^{\circledR}$ tablet], (in Japanese). 2012:23-24 Dainippon Sumitomo Pharma Co, Ltd. [www.info.pmda.go.jp/downfiles/ph/ PDF/400093_1129008F1039_2_04.pdf].

12. JP KK: Interview form [Risperdal ${ }^{\circledR}$ tablets \& OD tablets \& fine granules \& oral solution], (in Japanese). 2012:24-33. Janssen Pharmaceutical K.K. [http://www.info.pmda.go.jp/go/pack/1179038C1027_1_26/]

13. Company ELa: Interview form [Zyprexa ${ }^{\circledR}$ ], (in Japanese). 2012:106-113. Eli Lilly Japan K.K. [http://www.info.pmda.go.jp/go/pack/1179044F4028_1_17/]
14. Kamijima K, Nakamura J, Tsuboi K, Higuchi T: Review of the efficacy and safety of paroxetine - from the results of post-marketing surveillance. Japanese Journal of Clinical Psychopharmacology 2007, 10:1045-1061.

15. Omori T, Hiramatsu M, Asami Y, Morimasa M, Yamashita N, Houzawa H, Terasawa H, Hasegawa H: Safety and efficacy of J Zoloft ${ }^{\circledR}$ tablets in actual clinical settings: first interim report - findings from drug use result survey and specific drug use result survey for panic disorder. Japanese Journal of Clinical Psychopharmacology 2012, 15:561-573.

16. Brewerton TD: Antipsychotic agents in the treatment of anorexia nervosa: neuropsychopharmacologic rationale and evidence from controlled trials. Curr Psychiatry Rep 2012, 14:398-405.

doi:10.1186/1744-859X-12-7

Cite this article as: Okita et al:: Tandospirone, a 5-HT1A partial agonist is effective in treating anorexia nervosa: a case series. Annals of General Psychiatry 2013 12:7.

\section{Submit your next manuscript to BioMed Central and take full advantage of:}

- Convenient online submission

- Thorough peer review

- No space constraints or color figure charges

- Immediate publication on acceptance

- Inclusion in PubMed, CAS, Scopus and Google Scholar

- Research which is freely available for redistribution 